\title{
Research on Adaptive Controller for Variable Polarity Plasma Arc Welding Power Supply with High-Frequency
}

\author{
Yang Zhou ${ }^{1}$, Bojin $\mathrm{Qi}^{1, *}$, and Minxin Zheng ${ }^{1}$ \\ ${ }^{1}$ School of Mechanical Engineering and Automation, Beihang University, 100191 Beijing, P. R. China
}

\begin{abstract}
Aiming at the problem of output current waveform distortion caused by superimposed high frequency components in the output current of varying polarity of VPPAW power supply. A novel adaptive PID controller with automatic switching is introduced in this paper, and the controller is established based on the evolution path of the operating point of the VPPAW power supply. Compared with the traditional linear PID controller, the adaptive controller proposed in this paper is very suitable for the control of arc load with strong dynamic characteristics and can effectively improve output waveform of the welding system.
\end{abstract}

\section{Introduction}

In past decades, control system of variable polarity plasma arc welding (VPPAW) power supply has great development in control structure, control means and control laws [1], [2]. The VPPAW has the advantages of small welding defects, good cathode cleaning effect, strong penetration ability and small deformation of workpiece. Due to the complexity of welding process, the system model changes with the operating point of the welding process. Therefore, it is difficult to achieve accurate and stable control for VPPAW system with strongly nonlinear by using conventional linear control method [3-5].

The topology of the VPPAW power supply with high frequency designed in this paper is mainly composed of variable polarity base power supply and Pulse DC power supply, where these two power supply has external characteristics of constant current, so the two DC power supply has a feedback circuit. In the case of arc load, the high frequency overlay area of output current will show obvious current drop phenomenon. Therefore, the main purpose of this article is to design a controller with adaptive control capabilities to achieve optimal control for pulse DC power. Makes the control system adapt to the dynamic changes of the model and improves the stability of the welding power output waveform [6-10]. In order to solve this problem, this paper designs a PID controller with adaptive capability based on the research of output characteristics of plasma welding power source under different working modes. Finally, the reliability of the adaptive controller proposed in this paper is established and verified by hardware and Simulink simulation experiments.

\section{Design of VPPAW power supply}

\subsection{Main circuit and control system structure}

The VPPAW power supply main circuit and control system structure shown in Figure 1.

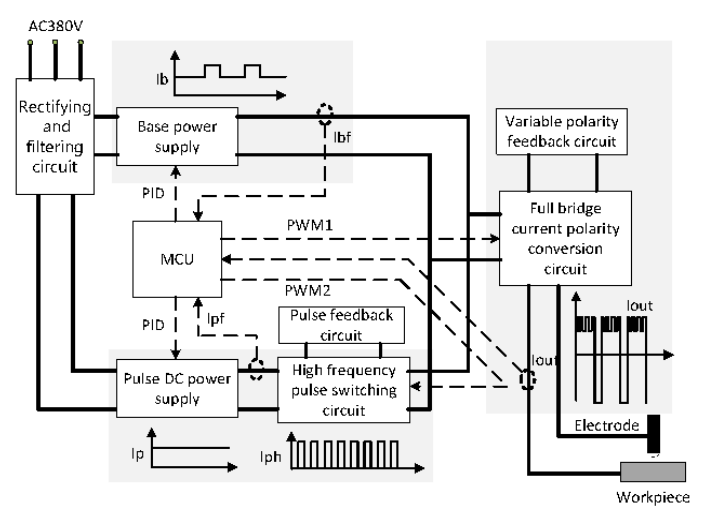

Fig. 1. The main circuit and control structure of VPPAW

The main components of the circuit are as follows:

- Base power supply: $I b(0 \sim 500 A)$.

- Full bridge current polarity conversion circuit: Polarity of superposition output waveform $I_{\text {out }}, P W M 1$ $(0 \sim 100 \mathrm{~Hz})$.

- Pulse DC power supply and High frequency pulse switching circuit: generate a constant current output Ip:0 100A and a duty cycle ultrasonic pulse square wave current Iph: $0 \sim 100 A, P W M 2$ $(0 \sim 100 \mathrm{kHz})$, respectively.

- Pulse feedback circuit and Variable polarity feedback circuit: IGBT protection and power recovery.

- MCU: Welding process control, parameter setting( $I b$, 
Ip , PWM1, PWM 2 ), welding process information feedback.

- Rectifying and filtering circuit: The input of the VPPAW power supply.

\subsection{Output waveform control}

The closed-loop control of the welding power supply designed in this paper is achieved by the combination of digital circuits (the MCU controller) and analog circuits. Where the MCU controller realizes the setting of the target value, and the analog circuit mainly realizes the closed-loop adjustment of the output value to tracking of the target value. For example, to adjust $I p$. First, write the given value to the lower computer of the MCU through the touch screen PC software. Then, the MCU converts the digital value of the given value into an analog level Ipg through the DAC conversion, and input with the feedback voltage value Ipf to PID controlling unit consists of op amp, SG3525AN integrated circuit, and the pulse width of the output PWM is adjusted to adjust the output current.

The main types of variable polarity output waveforms are shown in Figure 2.

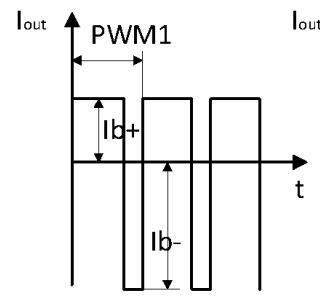

(A)

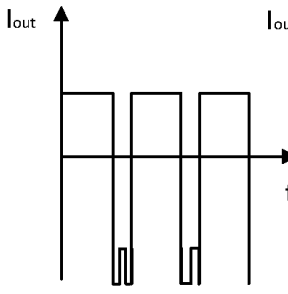

(C)

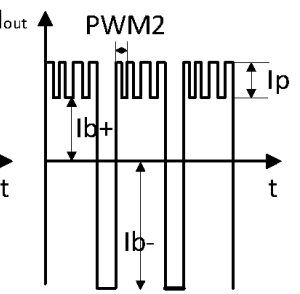

(B)

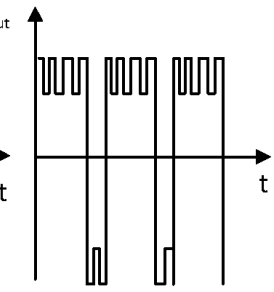

(D)
Fig. 2. Variable polarity output waveforms

Where, A, B, C, and D indicate non-superimposed, positively-superposed, negative-superposed, and fullysuperimposed output modes, respectively. And the parameters $I b+$ (Positive base current.), $I b-$ (Negative base current), Ip, PWM1 and PWM2 in Figure 2 can be adjusted in real time on the touch screen.

\section{Closed loop control of VPPAW power supply based on linear PID}

The closed loop control system designed in this paper is a pulse width modulation circuit based on SG3525AN, which can adjust the pulse width $(0 \sim 44 \%)$ of the PWM signal with fixed frequency $(20 \mathrm{kHz})$ to control the output of the Pulse DC power supply and the Base power supply in Figure 1.

\subsection{Structure of Closed-loop Control Circuit}

The schematic diagram of the closed-loop control structure circuit is shown in Figure 3.

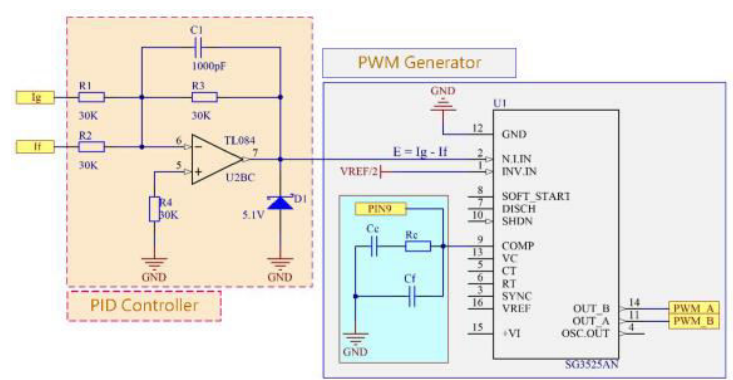

Fig. 3. The schematic diagram of the closed loop control circuit based on SG3525AN

In Figure 3, I $g$ is that the set value, If is the feedback value measured by the Hall sensor, $E$ represents the control quantity, PWMA, PWMB represent a pair of complementary PWM output waveforms. The orange area indicates the PID controller, in this figure, there is only one Proportional part, and the main function is to adjust the response time of the system, where the equivalent parameters are $\mathrm{P}=1, \mathrm{I}=0$, and $\mathrm{D}=0$. The gray area is the PWM generator. The blue area is used to adjust the response speed of the pulse width.

\subsection{Working principle of closed-loop control circuit}

The working principle of closed loop control Structure in Figure 3 is shown below:

- The PID controller output the control quantity $E$ in Orange Area according to the error value of $I g$ and $I f$.

- The PWM generator adjusts the pulse width of the PWM waveform in real time based on the output value of the PID controller and outputs it from the PWMA and PWMB to the drive circuit.

- In order to ensure the stability and safety of the welding power supply, we can adjust the RC circuit parameters in the blue region, to change the response speed of the circuit for the changes of the control quantity $E$. An excessively fast response speed can lead to system hardware failures, such as transformer bias, but too slow response will reduce the output current quality.

Therefore, choosing a proper combination of parameters can ensure a stable output of the system to some extent.

\subsection{Analysis of control results based on linear PID controller}

Obviously the control system shown in Figure 3 is a linear controller with deterministic parameters. However, the arc load is a dynamic non-linear load, and the arc load changes with the welding work point. Next, we will use VPPAW power supply designed in this 
paper to conduct welding experiments on a $10 \mathrm{~mm}$ thick pure Aluminum plate. The welding parameters are shown in Table 1, where $R c=510 \Omega, C c=104$, $C f=102$.

Table 1. Welding parameters of VPPAW

\begin{tabular}{|c|c|c|c|c|c|c|c|c|}
\hline \multirow{4}{*}{$\begin{array}{c}\text { Dat } \\
\text { a }\end{array}$} & \multicolumn{8}{|c|}{ Welding Parameters } \\
\hline & \multicolumn{4}{|c|}{$\mathrm{Ib}$} & \multicolumn{4}{|c|}{ Ip } \\
\hline & \multirow{2}{*}{$\begin{array}{c}\mathrm{Ib} \\
+\end{array}$} & \multirow{2}{*}{$\begin{array}{c}\mathrm{Ib} \\
-\end{array}$} & \multicolumn{2}{|c|}{ PWM2 } & \multirow{2}{*}{ Ip } & \multicolumn{2}{|c|}{ PWM1 } & \multirow{2}{*}{ Mode } \\
\hline & & & $\mathrm{Fb}$ & $\mathrm{Db}$ & & $\mathrm{Fp}$ & $\mathrm{Dp}$ & \\
\hline No & 10 & 10 & 50 & 80 & 50 & $20 \mathrm{~K}$ & 50 & \\
\hline 1 & A & $\begin{array}{l}0 \\
\mathrm{~A}\end{array}$ & $\mathrm{~Hz}$ & $\%$ & A & $\mathrm{Hz}$ & $\%$ & ve \\
\hline
\end{tabular}

The welding output waveforms are shown in Figure

4.

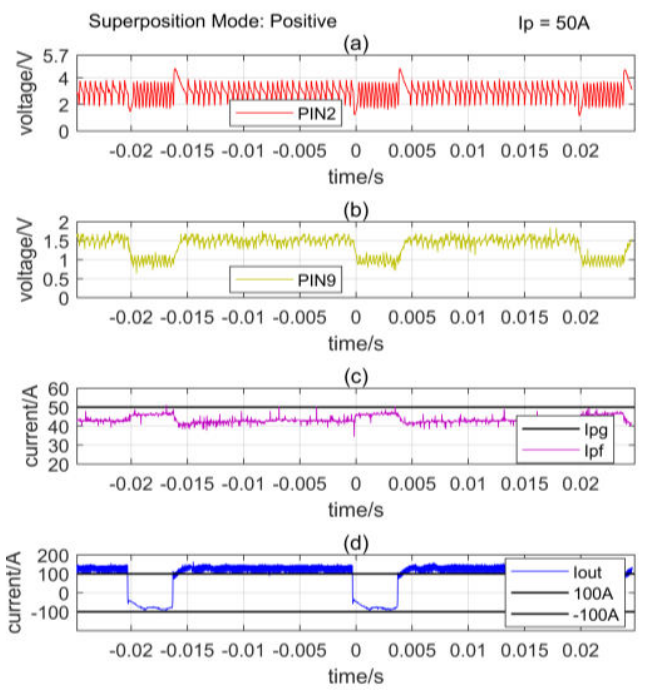

Fig. 4. The welding output waveforms

Where, the Figure 4. (a) and (b) are the input and output signals of pin 2 and pin 9 of SG3525 respectively, and the Figure 4. (c) represent the set-points (the Black Curve) and feedback values (the Purple Curve), respectively. Figure 4. (d) shows the total output current waveform of the welding power supply formed by the superposition of the base-polarized current $I b$ and the ultrasonic pulse square-wave current $I p$.

According to Figure 4. (c), we find that there is always a difference between the actual output value $I g f$ and the set value Ipg, and this difference changes with the superposition mode. The main reason for this problem is that the system model (or the model of the load) changes with the operating mode during the welding process. Therefore, the linear PID controller with deterministic parameters will be difficult to obtain stable output performance for the control of the welding process.

\section{Design of adaptive controller}

This section mainly introduces the design method and working principle of the adaptive controller.

\subsection{Establishing system models at different operating points}

Combination with the experimental results shown in Figure 3, and combine the experimental results shown in Figure 4 and the parameter setting of variable polarity drive waveform PWM1 in Table 1, we can divide the output current waveform of a pulsed power supply in Figure 4. (c). As shown in Figure 5

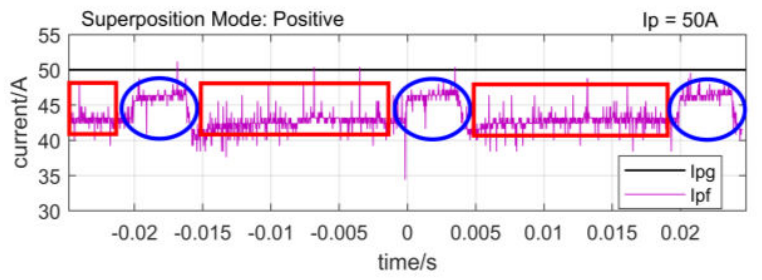

Fig. 5. The input and output curves of the pulsed DC power supply

In Figure 5, the purple curve represents the output feedback value Ipf , which jumps between two levels, where the direction of the jump is opposite to the superposition direction. In the superposition region, the output current transitions downwards, and in the nonsuperimposed region, the output current rises upward. For example, In Figure 5, the red rectangular region represents the superimposed region and the blue oval region represents the un-superimposed region. However, during the entire welding process, the set value of the power supply is always $50 \mathrm{~A}$, and the change of the working point is equivalent to the change of the load model. Therefore, the dynamic change of the feedback signal reflects the characteristics of the arc load at different operating points.

According to the results of the welding experiment in Figure 4, when the welding parameters are set in Table 1 , it determines what type of pulse waveform the output of the pulse DC power will be superimposed with the base value in which time zone. Therefore, the evolution path of the output current model is determined. There is no need to consider the randomness of the model due to the change of the working side.

Next, we will establish the model of the Pulsed DC Power in different superposition modes.

- Non-superposition mode:

Fig. 6 shows the open loop step response curve of the Pulse DC Power Supply without superposition.

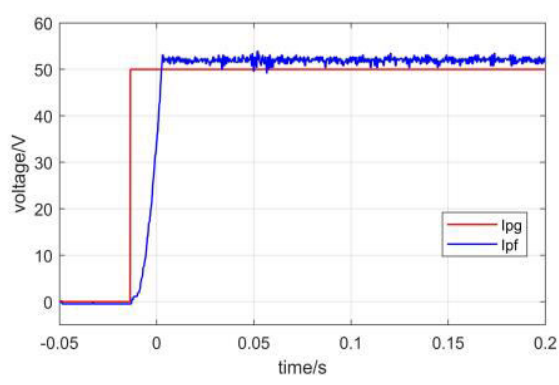

Fig. 6. The step response curve without superposition 
Where, the red curve shows the set value Ipg and the blue curve shows the response value Ipf . Then, using the SystemIdentification toolbox of MATLAB to identify the step model of the current system.

$$
G(s)_{1}=\frac{133}{s+127.4} \cdot e^{-0.0029 s}
$$

\section{- Positive-superposition mode:}

Fig. 7 shows the open loop step response curve of the pulse DC power supply with Positive-superposition.

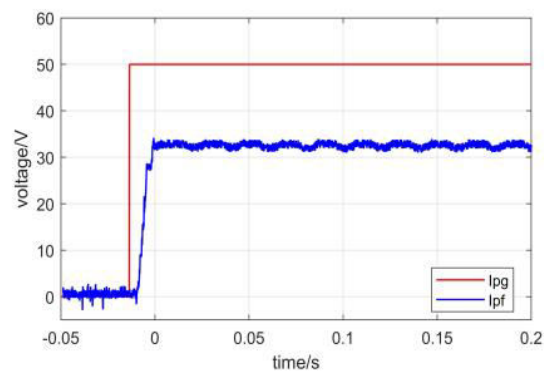

Fig. 7. The step response curve with Positive-superposition

After model identification, the open-loop transfer function of the system model is (2).

$$
G(s)_{2}=\frac{170.5}{s+261.2} \cdot e^{-0.0027 s}
$$

- Negative-superposition mode:

Fig. 8 shows the open loop step response curve of the pulse DC power supply with Negative-superposition.

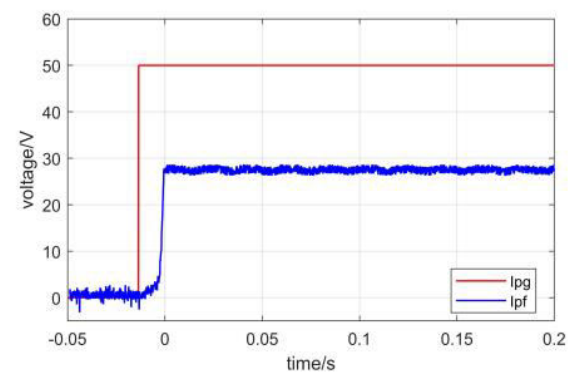

Fig. 8. The step response curve with Negative-superposition

Similarly, we can get the open-loop transfer function of the system model as (3).

$$
G(s)_{3}=\frac{69.23}{s+125.1} \cdot e^{-0.0021 s}
$$

\subsection{Design of adaptive controller}

According to the three open-loop step response experiments in Section 4.1, we have obtained the openloop transfer function model of the system in three different modes (1), (2) and (3). Where, the system model of full- superposition mode can be represented by (2) and (3) to reflect the nonlinearity of the system.

Based on the above three models (1) (3), a closedloop control system based on PID controller is established, and the PID-Tuner toolbox of MATLAB are used to calculate the parameters of the three PID controllers separately. As shown in Table 2.

Table 2. The parameters of the PID controller for the three models

\begin{tabular}{|c|c|c|c|c|}
\hline \multicolumn{2}{|c|}{ Model } & $P I D_{\text {Nos. }}$ & $P I D_{\text {Posi. }}$ & $P I D_{\text {Neg. }}$ \\
\hline \multirow{4}{*}{$\begin{array}{c}\text { Paramete } \\
\text { rs }\end{array}$} & $K_{p}$ & 2.86 & 6.07 & 7.81 \\
\cline { 2 - 5 } & $K_{I}$ & $5.68 \times 10^{2}$ & $3.09 \times 10^{3}$ & $2.02 \times 10^{3}$ \\
\cline { 2 - 5 } & $K_{D}$ & $1.36 \times 10^{-3}$ & $1.42 \times 10^{-3}$ & $3.65 \times 10^{-3}$ \\
\hline
\end{tabular}

As long as the parameters of the welding process are determined, the evolution path of the PID parameters in is also determined. Thus, the structure of the adaptive controller designed in this paper is constructed.

For example, the parameter configuration shown in Table 1, the evolution path of the adaptive controller is shown in Figure 9.

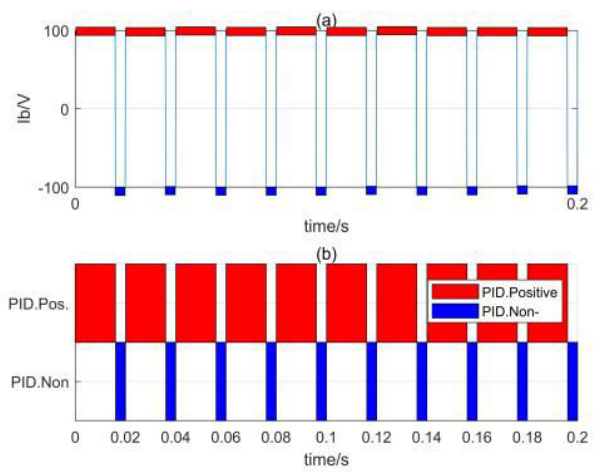

Fig. 9. The evolution path of the adaptive controller

Where, the Figure 9. (a) represents the base value of the polarity waveform, the frequency is $50 \mathrm{~Hz}$, the positive pulse width duty cycle is $80 \%$, and the Figure 9 . (b) shows that the evolution path of the PID controller varies with the pulse width. And in Table 1, the superposition mode of the ultrasonic pulse square-wave current supply is defined as positive, so the controller in the positive pulse width area is $P I D_{\text {Posi. }}$. (the red rectangle block), and the negative pulse width area is $P I D_{\text {Non. }}$ (the blue rectangle block).

\section{Analysis of experimental results}

This chapter mainly verifies the control performance of adaptive PID control strategy (proposed in Section 4.2) for dynamic models by Simulink simulation.

The simulation control system shown in Figure 10.

The brown area in Figure 10. (a) indicates the parameter setting module. The cyan area in Figure 10. (b) represents the structure of the adaptive PID controller, and the green area is a dynamic model consisting of the Positive-superposition mode and the Non-superposition mode, where the PID controllers and models change with the pulse width of PWM1. Figure 10. (c) has the same structure as Figure 10.b except that 
the controller is a linear controller and the controller parameters do not change with PWM1.

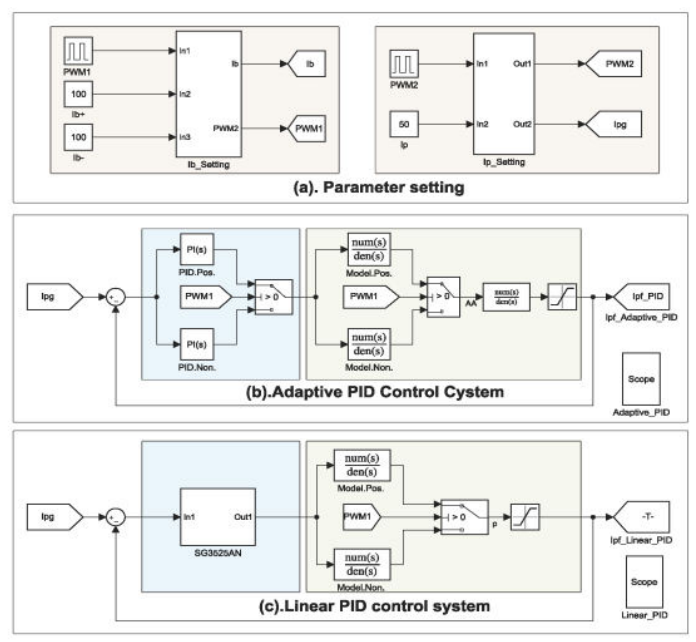

Fig. 10. Simulation control system

Assume that the simulation parameters are consistent with Table 1, and the simulation results are shown in Figure 11.
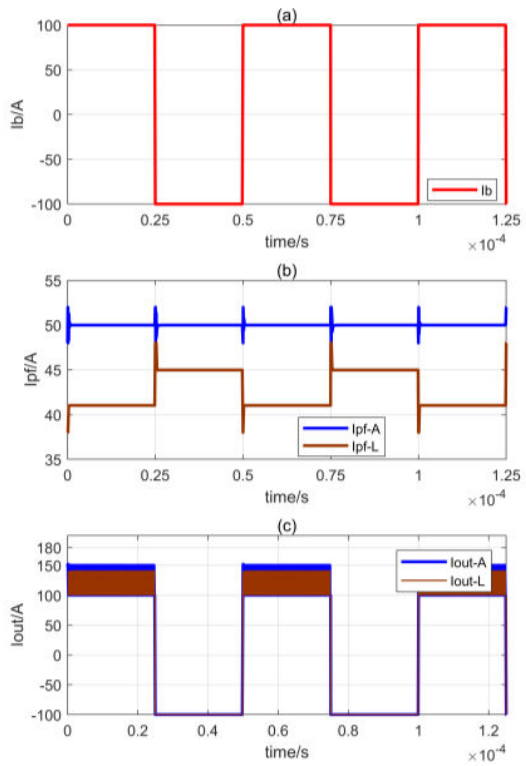

Fig. 11. Simulation results

Where, the Figure 11. (a) shows the set value of the base value of variable polarity $I b+=100 A, I b-=-100 A$. Figure 11. (b) represents the current feedback with the control of two controllers as shown in Figure 10. $(b \sim c)$ for the Pulsed DC power supply, where the blue curve is the control result of the adaptive PID controller, always keep track for the set value Ipg $=50 A$ with a small overshoot at the switching point, the brown curve is the control result of the linear PID controller, and is the same as the experimental result as shown in Figure 5. Therefore, the Ipf $-L$ curve not only shows that the linear PID has poor control performance for the dynamic model studied in this paper, and it proves that the mathematical models (1)-(3) established in Chapter 4.1 of this paper are reliable. At the same time, it also proves that the simulation control system established in this chapter (as shown in Figure 10) can well reflect the dynamic characteristics of the actual system.

The Figure 11. (c) shows the variable polarity output of the variable polarity plasma welding (VPPAW) power supply, the blue curve represents the output curve with the control of the adaptive PID controller, and the brown curve represents the output current with the control of the linear PID control. It can be seen that only the control output waveform based on the adaptive PID control can well achieve the target value set in Table 1 .

Therefore, the adaptive PID controller designed in this paper can improve the control performance and the output waveform of VPPAW welding power supply.

\section{Conclusion}

According to experimental results and analysis, we conclude that the adaptive PID controller designed in this paper can achieve accurate and stable control for the VPPAW welding power supply with dynamically changing of working points. The results of comparative experiments show that the adaptive controller can improve the control performance for the control of the VPPAW welding power supply superimposed with high frequency current, it has strong robustness, and improves the ability of the control system to adapt to the dynamic changes of the controlled system. Therefore, the adaptive PID controller designed in this paper is reliable for improving the control performance of VPPAW welding power supply.

\section{References}

1. Liu Z.M., "Review: Plasma arc welding: Process variants and its recent developments of sensing, controlling and modeling", Journal of Manufacturing Processes, vol. 23, pp. 315-28. 2016.

2. Wu C.S., W.L., R.W.J., "Technical paper: Plasma arc welding: Process, sensing, control and modeling", Journal of Manufacturing Processes, vol. 16(1), pp. 74-86. 2014.

3. Song S., Chen H., Lin T., Wu D., Chen S.B, "Penetration state recognition based on the doublesound-sources characteristic of VPPAW and hidden Markov Model", Journal of Materials Processing Technology, vol.234, pp. 33-44, 2016.

4. Hong H. "Investigation on droplet momentum in VPPA-GMAW hybrid welding of aluminum alloys", International Journal of Advanced Manufacturing Technology, vol. 86(5-8), pp. 23019, 2016.

5. Morgan., Abdul A.A.Al-Mufadi., Fahad A., "A novel method to improve weld uniformity by automatic control3 of current intensity", American journal of engineering and applied sciences, vol. $8(1)$, pp.17-26,2015. 
6. Algreer M., Armstrong M., Giaouris D., "Adaptive PD+I Control of a Switch-Mode DC-DC Power Converter Using a Recursive FIR Predictor", IEEE Transactions on Industry Applications, vol. 47(5), pp. 2135-44. 2011.

7. Shirazi M., Zane R., Maksimovic D., "An Autotuning Digital Controller for DC-DC Power Converters Based on Online Frequency-Response Measurement", IEEE Transactions on Power Electronics, vol. 24(11), pp.2578-88,2009.

8. Liu Y.F., Meyer E., Liu X., "Recent Developments in Digital Control Strategies for DC/DC Switching Power Converters", IEEE Transactions on Power Electronics, vol. 24(11), pp. 2567-77,2009.

9. Guo L., Hung J.Y., Nelms R.M., "Evaluation of DSP-Based PID and Fuzzy Controllers for DC-DC Converters", IEEE Transactions on Industrial Electronics, vol. 56(6), pp. 2237-48, 2009.

10. Corradini L., Orietti E., Mattavelli P., Saggini S., "Digital Hysteretic Voltage-Mode Control for DCDC Converters Based on Asynchronous Sampling", IEEE Transactions on Power Electronics, vol. 24(1), pp.201-11, 2009. 